\title{
Immunochemical Analysis and Immunogenicity of the Type II Group B Streptococcal Capsular Polysaccharide
}

\author{
Dennis L. Kasper, Carol J. Baker, Bridget Galdes, Ewa Katzenellenbogen, \\ and Harold J. Jennings, Channing Laboratory, Brigham and Women's \\ Hospital, and the Division of Infectious Diseases, Beth Israel Hospital, \\ Harvard Medical School, Boston, Massachusetts 02115; Department of \\ Pediatrics, Microbiology, and Immunology, Baylor College of Medicine, \\ Houston, Texas 77025; Division of Biological Sciences, National Research \\ Council of Canada, Ottawa, Ontario, Canada
}

\begin{abstract}
A B S T R A C T The relationship between group B streptococcal (GBS) type-specific antisera and the type II-specific polysaccharide is evaluated from a structural and immunologic viewpoint. Although all GBS type-specific polysaccharides are composed of the same monosaccharides, the type II antigen is more complex structurally and contains these sugars in a molar ratio different from the other antigens. Type II polysaccharide has two side chains. One contains only sialic acid and is less susceptible to acid cleavage than sialic acid residues found on types III, Ia, and Ib polysaccharides. The other side chain is composed of galactose as the only sugar. Immunochemical studies demonstrate that the type II polysaccharide has several immunodeterminants. One of these determinants is likely to be the side-chain galactose, while sialic acid appears to comprise part of another immunodeterminant, more complex than sialic acid alone. A series of cross-reactions is demonstrated between the type II native antigen and antisera to serotypes Ia, III, and Ib by a sensitive radioactive antigen-binding assay, which account for additional, complex immunodeterminants. The strongest of these cross-reactions is with type Ia antiserum and the weakest with $\mathrm{Ib}$ antiserum. Since Ia and Ib polysaccharides differ in only one linkage, these findings suggest that the trisaccharide $\beta \mathrm{D}$ $N$ - acetyl - glucosamine - $p(1 \rightarrow 3) \beta D$ - galactose - $p(1 \rightarrow$ 4) $\beta \mathrm{D}$-glucose- $p\{[\beta \mathrm{D}-\mathrm{GlcNAcp}(1 \rightarrow 3) \beta \mathrm{D}-\mathrm{Gal} p(1 \rightarrow$ 4) $\beta \mathrm{D}-G l c p]\}$ is the likely common site responsible for the interaction of the type II native polysaccharide
\end{abstract}

Received for publication 29 October 1982 and in revised form 7 March 1983. and type Ia antiserum. Another cross-reaction is observed between type III antiserum and type II native antigen. Inhibition studies indicate that the most likely cross-reactive determinant in this case is $[\beta \mathrm{D}-\mathrm{Gal} p(1 \rightarrow$ 4) $\beta \mathrm{D}-$ GlcNAcp . Type II polysaccharide has been utilized in a human vaccine trial to test safety and immunogenicity. The polysaccharide is highly immunogenic, inducing an antibody response in $95 \%$ of recipients, and nontoxic, with side-effects confined to minimal local reactions. Despite the cross-reactions observed between type-specific antigens and antibody prepared by immunization of rabbits with whole bacteria, which suggest shared immunodeterminants, similar cross-reactions were not detected in human sera after immunization with purified type II polysaccharide.

\section{INTRODUCTION}

For more than a decade, group B streptococci (GBS) ${ }^{1}$ have been a leading cause of neonatal sepsis in the United States (1). Among the five serotypes of group B streptococci, type II strains, which account for $\sim 15 \%$ of all infant infections, have been isolated only rarely from infants with meningitis $(2,3)$. Among GBS isolates from adults with meningitis, however, type II strains predominate (3).

\footnotetext{
${ }^{1}$ Abbreviations used in this paper: GBS, group B streptococci; Galp, galactose; GlcNAcp, $N$-acetyl-glucosamine; Glcp, glucose; $K_{\mathrm{av}}$, stationary volume of a given solute species available for diffusion; NeuNAcp, sialic acid; RABA, radioactive antigen-binding assay.
} 
Antibodies directed against the type II capsular polysaccharide have been shown to be protective in mice challenged with homologous organisms and to be bactericidal with polymorphonuclear leukocytes and complement in an opsonophagocytic assay $(4,5)$. In addition, opsonic antibody to type II organisms has been reported to be absent in the sera of mothers and neonates who subsequently develop type II infection (6).

A structure has been proposed by Jennings et al. (7) for the native type II GBS polysaccharide. Like the other type-specific GBS polysaccharides, the type II antigen is composed of a repeating unit of five monosaccharides. The molar ratio of these sugars differs in the type II polysaccharide from the others. The structure also is more complex. The repeating unit of this polysaccharide is represented in Fig. 1. The type II antigen has two side chains, each a monosaccharide, in contrast to the other type-specific polysaccharides that have one side chain composed of several sugars. Sialic acid directly attaches to the backbone of the type II antigen.

Preliminary immunochemical studies of the type II polysaccharide performed by Lancefield (8) and Freimer (9) indicated that this antigen had an acid labile determinant that was part of a more complete immunodeterminant. The present study was designed to further characterize the immunodeterminants of the type II polysaccharide based on a knowledge of its structure, to analyze observed cross-reactions of the antigen with animal and human sera directed against the type-specific polysaccharides, and to evaluate the safety and immunogenicity of this antigen in humans.

\section{METHODS}

Strains. Prototypic strains of GBS, representing each of the five serotypes and the group $B$ variant strain (devoid of type-specific antigen), originally were supplied by the late Dr. Rebecca Lancefield, Rockefeller University. These strains are designated 090 (type Ia), H36B (type Ib), A909 (type Ic), 18RS21 (type II), D136C (type III), and 090R (group B). In addition, type III antigens were prepared from type III strain $\mathrm{M} 732$, an isolate from an infant with meningitis. Lyophilized strains were rehydrated with Todd-Hewitt broth (Difco Laboratories, Inc., Detroit, MI) streaked onto $5 \%$ blood agar plates and incubated overnight at $37^{\circ} \mathrm{C}$.

Rabbit antisera. New Zealand White rabbits were immunized with formalin-killed whole bacterial preparations of the prototypic strains of GBS according to the method of McCarty and Lancefield (10). Unabsorbed antisera were utilized for further definition of cross-reacting antigenic determinants, although the standard procedure for preparation of types Ia- and Ib-specific antisera involves absorption with the alternate bacterial strain $(11,12)$. The sera used in these latter studies were prepared with formalin-fixed organisms grown under conditions of neutral $\mathrm{pH}(13,14)$.

Antigen preparation. Native (containing sialic acid) and core (lacking sialic acid) polysaccharides of type II GBS were extracted and purified by methods described for the type Ia and III polysaccharides (7, 13-15). Carbohydrate composition and structural analysis of the type Ia $(14,15)$, Ib (15), and III $(13,16)$ polysaccharides are described in detail elsewhere and summarized in Fig. 1. These antigens contain no detectable protein or nucleic acid. Furthermore, by gas-liquid chromatography no rhamnose, an indicator monosaccharide for contamination with group-specific antigen, was found in any preparation. Type Ia, Ib, and III native and core antigens were extracted and characterized as previously described (13-16).

Chemical modification of polysaccharides. The backbone of the type III antigen used in inhibition studies was prepared by the removal of the terminal $\beta \mathrm{D}$-galactopyranosyl residues from the type III core antigen as previously described (16). In this procedure the primary hydroxyl groups of the terminal $\beta \mathrm{D}$-galactopyranosyl residues were oxidized selectively with galactose oxidase to aldehyde groups. Following basic elimination, these terminal residues were removed by short treatment with mild acid.

The degalactosylated type Ia and Ib antigens (performed after removal of sialic acid) previously shown to be identical structurally (15), were prepared by the controlled periodate oxidation of their respective core antigens (15). In this reaction the terminal $\beta \mathrm{D}$-galactopyranosyl residues were oxidized selectively, and the remnants of these residues removed by reduction and mild acid treatment of the oxidized core antigens. The common backbone of the type Ia and Ib antigens were prepared by deamination of the $\boldsymbol{N}$-deacetylated type Ia or Ib core antigens (15). Accomplishment of all these structural modifications was confirmed by methylation analysis and ${ }^{13} \mathrm{C}$ nuclear magnetic resonance spectroscopy $(15,16)$. The structures of these modified antigens are shown in Fig. 1. The group B polysaccharide was prepared by the method of Curtis and Krause (17) and contained $60 \%$ rhamnose. The $K_{\mathrm{av}}$ for the native and core type II polysaccharides was determined on a $2.6 \times 90-\mathrm{cm}$ column of Sepharose 4B (Pharmacia Fine Chemicals, Piscataway, NJ) equilibrated in $0.05 \mathrm{M}$ Tris $\mathrm{HCl}$ buffer, $\mathrm{pH}$ 7.3. The $K_{\mathrm{av}}$ $=(\mathrm{Ve}-\mathrm{Vo}) /(\mathrm{Vt}-\mathrm{Vo})$, where $\mathrm{Ve}=$ elution volume, $\mathrm{Vt}$ $=$ bed volume, and Vo $=$ void volume. The $K_{\mathrm{av}}$ represents the fraction of the stationary volume of a given solute species available for diffusion and defines solute behavior independently of bed dimensions and packing (18). Therefore, this constant can be used for comparative estimates of molecular size of polysaccharides (19).

Serological methods. Capillary precipitin tests were performed by the method of Lancefield $(11,12)$. Double diffusion in agar was prepared as described by Ouchterlony (20). Immunoelectrophoresis was performed by the method of Scheidegger (21) under conditions previously published for the type III polysaccharide (22).

Antibodies to the type Ia and III polysaccharides in sera from human volunteers were quantified by a radioactive antigen-binding assay (RABA) $(23,24)$. Antibody to the type II polysaccharide was quantified by a modification of the RABA, analyzing the supernatant rather than the antigen pellet. Instead of determining the percentage of radiolabeled antigen bound by counting the precipitate of a mixture of antiserum plus labeled antigen after washing with $50 \%$ ammonium sulfate, a supernatant aliquot was obtained with a highly reproducible pipette (Eppendorf) and counted. The correlation coefficient (method of least squares) was 0.98 between the percent antigen remaining in the supernatant in the modified RABA and the $\log _{10}$ antibody content, as determined in the quantitative precipitin assay.

To study cross-reactions between the intrinsically labeled type II native polysaccharide ( $200 \mathrm{ng},{ }^{3} \mathrm{H}$-labeled) and var- 
Structural modifications of group B streptococcal polysaccharide antigens

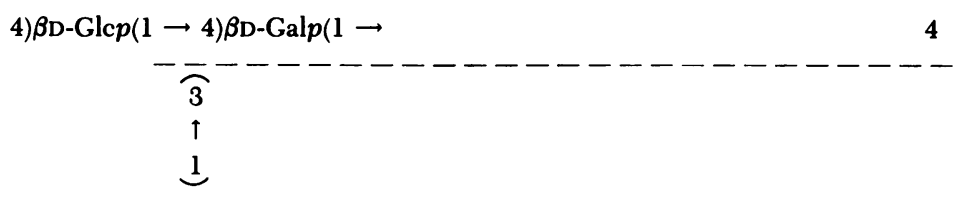

I $a$

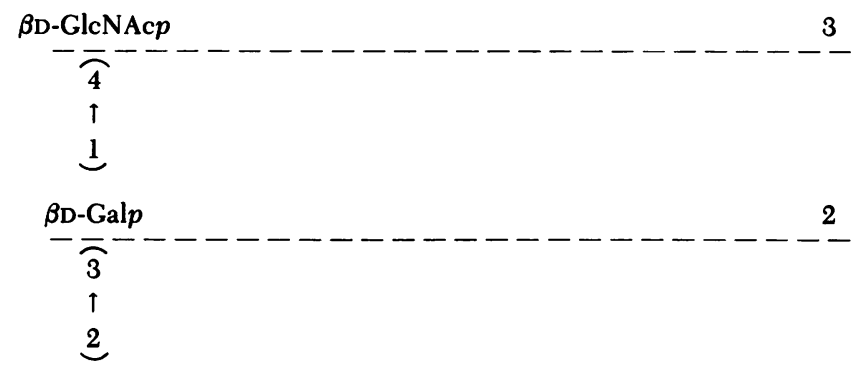

$\alpha$ D-NeuNAcp

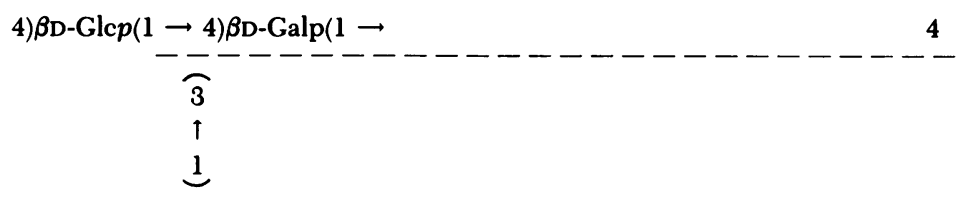

Ib

$\beta \mathrm{D}-\mathrm{GlcNAcp}$

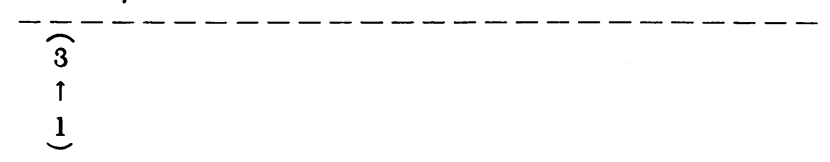

$\beta \mathrm{D}-\mathrm{Galp}$

2

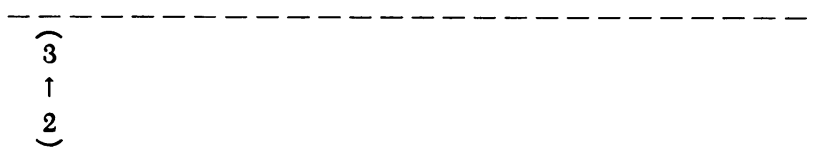

$\alpha \mathrm{D}-\mathrm{NeuNAcp}$

1

II

4) $\beta \mathrm{D}-\mathrm{GlcNAcp}(1 \rightarrow 3) \beta \mathrm{D}-\mathrm{Gal} p(1 \rightarrow 4) \beta \mathrm{D}-\mathrm{Glcp}(1 \rightarrow 3) \beta \mathrm{D}-\mathrm{Glcp}(1 \rightarrow 2) \beta \mathrm{D}-\mathrm{Gal} p(1 \rightarrow$

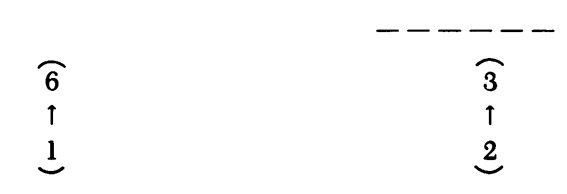

$\beta \mathrm{D}-\mathrm{Gal} p$

$\alpha$ D-NeuNAcp

1

4) $\beta \mathrm{D}-\mathrm{Glcp}(1 \rightarrow 6) \beta \mathrm{D}-\mathrm{GlcpNAcp}(1 \rightarrow 3) \beta \mathrm{D}-\mathrm{Gal} p(1 \rightarrow$

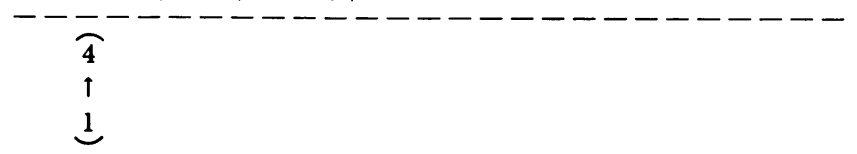

III

$\beta \mathrm{D}-\mathrm{Gal} p$

2

$--------------------$

6

$\uparrow$

2

$\alpha \mathrm{D}-\mathrm{NeuNAc} p$ 
ious antisera, competitive inhibition experiments were designed. The serotype Ia and III antisera, which demonstrated cross-reaction with the native type II polysaccharide, were diluted to a point previously determined to be near the top of the rapidly descending portion of a curve for the RABA. Inhibition studies were not performed with type Ib antiserum because the cross-reaction seen was weak. Competitive inhibition of the type II RABA was achieved by preincubating 250,500 , and $1,000 \mathrm{ng}$ of each serotype-specific polysaccharide with $0.05 \mathrm{ml}$ of serum at the predetermined dilution for $2 \mathrm{~h}$ at $25^{\circ} \mathrm{C}$. Data from these experiments are expressed as the calculated amount of antigen displaying $50 \%$ inhibition when compared with inhibition by the homologous unlabeled type II polysaccharide at an equivalent concentration.

Quantitative precipitin and quantitative precipitin inhibition studies were conducted as previously described (22).

Native type II GBS polysaccharide vaccine. Purified native type II polysaccharide was suspended in pyrogen-free phosphate-buffered saline at $100 \mu \mathrm{g} / \mathrm{ml}$. Bulk sterility tests were performed before final packaging. Animal toxicity and sterility testing of final packaged vaccine were completed successfully by Ms. Leslie Wetterlow under the direction of Dr. George Wright and Dr. George Grady at the Massachusetts State Laboratory Institute. This testing complied with the regulations of the Food and Drug Administration (Title 21 , section $\$ 610.11$ and $\$ 610.12$ ).

Human subjects. 20 adult volunteers received purified native type II polysaccharide vaccine. Immune status of subjects was unknown before vaccination. A single $50 \mu \mathrm{g}$ dose $(0.5 \mathrm{ml})$ was given subcutaneously in the deltoid region after written informed consent was obtained from each subject and after a preimmunization serum specimen was drawn. Local and systemic reactions following immunization were monitored for $48 \mathrm{~h}$. Serum specimens were obtained for antibody determinations at 4 wk and 2 yr after immunization for most subjects. These sera were tested for the development of antibody to native type Ia, II, and III polysaccharides by the RABA. Preimmunization and 4-wk postimmunization serum pairs were tested on the same day. All sera were tested in duplicate.

Paired sera from three volunteers who had received native type Ia polysaccharide vaccine and from 20 volunteers who had received the native type III polysaccharide vaccine $(25$, 26) were tested for the development of antibodies cross-reactive with the native type II polysaccharide. Sera were stored at $-70^{\circ} \mathrm{C}$ before testing.

The geometric mean antibody concentration and $95 \%$ confidence intervals were calculated for each group of subjects (26). Differences in concentrations of antibody in pre- and postimmunization sera of individuals were determined by the paired $t$ test (27).

\section{RESULTS}

Molecular size of type II antigens. The $K_{\mathrm{av}}$ of the type II native polysaccharide was 0.26 , while the $K_{\mathrm{av}}$
TABLE I

Quantity of Antibody to the Native Type II Polysaccharide in Unabsorbed Antisera to Various Serotypes of Group B Streptococcus

\begin{tabular}{lccccc}
\hline Sera made to strain of serotype & Ia & Ib & II & III & Group B \\
\hline $\begin{array}{l}\text { Quantity of antibody to } \\
\text { native type II } \\
\text { antigen }(\mu g / m l)\end{array}$ & 342.0 & 5.2 & $3,906.0$ & 83.0 & $<2$ \\
$\begin{array}{l}\text { Capillary precipitin } \\
\text { reaction with type II }\end{array}$ & - & - & $4+$ & - & - \\
$\begin{array}{l}\text { Capillary precipitin } \\
\text { with homologous } \\
\text { serotype or } \\
\text { serogroup antigen }\end{array}$ & $4+$ & $4+$ & $4+$ & $4+$ & $4+$ \\
\hline
\end{tabular}

of the type II core polysaccharide was 0.48 , indicating the larger size of the native polysaccharide.

Immunologic definition of the native and core polysaccharides of type II GBS. Capillary precipitin tests with the native or core type II polysaccharide and the various serotype-specific antisera or group B-specific serum demonstrated type II specificity (Table I). The standard methods of double diffusion in agar and capillary precipitation confirmed the type II specificity of both the native and modified antigens (Fig. 2). The native type II polysaccharide was more complete (well 1 ) as evidenced by spurring across the less complete core antigen (well 2) when both were precipitated with type II antiserum. Treatment of the native polysaccharide with $6 \%$ acetic acid for $2 \mathrm{~h}$ at $100^{\circ} \mathrm{C}$ resulted in the loss of the additional determinant, elimination of spurring, and identity with the core polysaccharide (well 3). Two percent acetic acid treatment failed to remove completely the acid labile determinant.

Immunoelectrophoresis of the native (lower well) and core (upper well) type II antigens demonstrated the net negative charge of the native antigen (Fig. 3). The core antigen did not migrate under the electrophoretic conditions used. These observations indicated the presence of a labile acidic component that the structural studies (7) have defined as sialic acid.

Multiple determinant specificities of antibodies in rabbit serotype II GBS antiserum directed toward the type II polysaccharide. Equivalence points were determined by quantitative precipitation for the native and core type II polysaccharide with a standard type

Figure 1 Structural modifications of types Ia, Ib, II, and III group B streptococcal polysaccharide antigens. 1 , native antigen, no modification; 2 , core antigen, sialic acid removed; 3 , degalactosylated antigen, side-chain galactose removed; 4 , backbone antigen, complete side chain removed. 


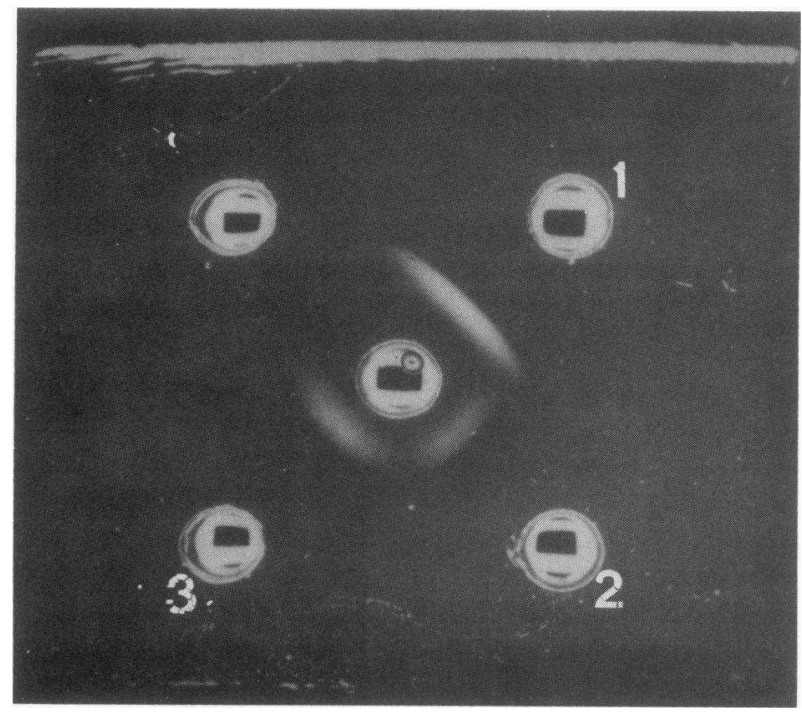

Figure 2 Double diffusion in agar of native type II group B streptococcal polysaccharide (well 1), core type II polysaccharide (well 2), and type II native polysaccharide treated with $6 \%$ acetic acid for $2 \mathrm{~h}$ at $100^{\circ} \mathrm{C}$ (well 3) vs. type II group B streptococcal antiserum (center well).

II antiserum. $1 \mathrm{ml}$ of type II antiserum was absorbed at equivalence with native type II antigen first at $25^{\circ} \mathrm{C}$ for $2 \mathrm{~h}$ and then at $4^{\circ} \mathrm{C}$ for $48 \mathrm{~h}$. After removal of the precipitate by centrifugation, varying amounts of core antigen were added to $50-\mu \mathrm{g}$ aliquots of the absorbed type II antiserum. After absorption, most of the antibodies that had reacted with the core antigen in unabsorbed type II antiserum had been eliminated (Fig. 4). However, in the converse experiment, after absorption of type II antiserum with an equivalence con- centration of core antigen, a substantial quantity of antibody remained in the serum that could be precipitated with the native type II polysaccharide (Fig. 4). These data were consistent with the results of double diffusion in agar experiments (Fig. 2) and specifically demonstrated the existence of two antibody populations in the type II group B streptococcal antiserum, as suggested by Lancefield and Freimer (8). One population was directed at the native type II antigen and the other at the core antigen.

A series of monosaccharide-inhibition studies was conducted to search for an immunodominant monosaccharide (Fig. 5). Galactose inhibited a maximum of $50 \%$ of the antibodies that precipitated with either the core or native antigen, but this degree of inhibition required a large excess of galactose $(>1,000$-fold) compared with the quantity of precipitating polysaccharide. This monosaccharide seemed unlikely to constitute a high-affinity immunodeterminant. The inhibition reaction by galactose was specific and dose related, however, while other monosaccharides failed to inhibit even at high concentrations.

A "pseudoinhibition" was observed when sialic acid was used as the inhibiting monosaccharide for precipitation with type II antiserum of either the native or core polysaccharide. The acidic nature of sialic acid caused the $\mathrm{pH}$ of inhibition tubes containing this sugar $(2-20 \mathrm{mg})$ to fall to 3.5 . When the $\mathrm{pH}$ was raised to 7.6 with $0.01 \mathrm{~N} \mathrm{NaOH}$, inhibition by sialic acid did not occur (Fig. $5 a$ and $b$ ).

Multiple cross-reactive antibodies in group B streptococcal type-specific antisera. The RABA provided a highly sensitive tool to determine the presence of antibodies directed to the native type II polysaccharide in unabsorbed rabbit sera raised to each of the type-

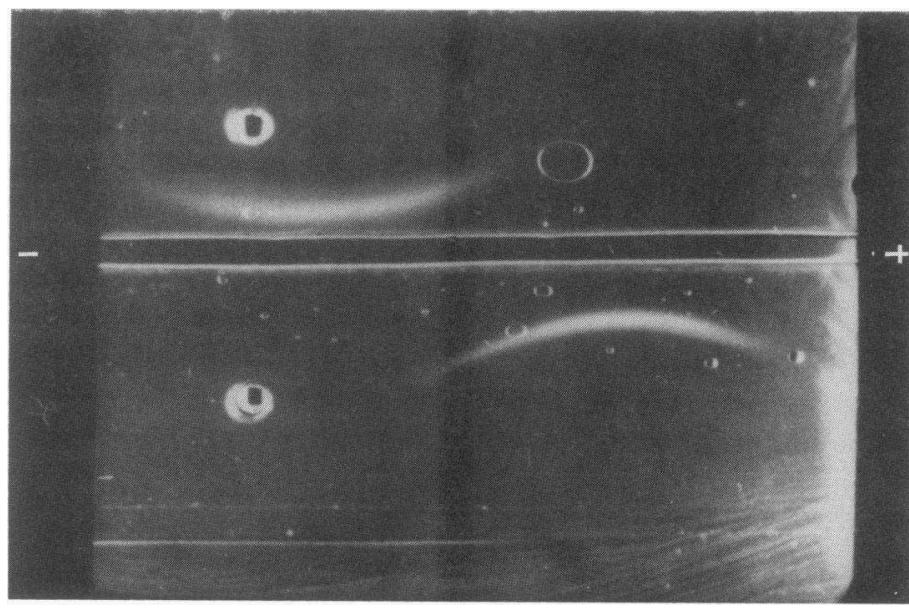

FIGURE 3 Immunoelectrophoresis of native type II group B streptococcal antigen (lower well) and core type II antigen (upper well) vs. type II group B streptococcal antiserum (trough). 


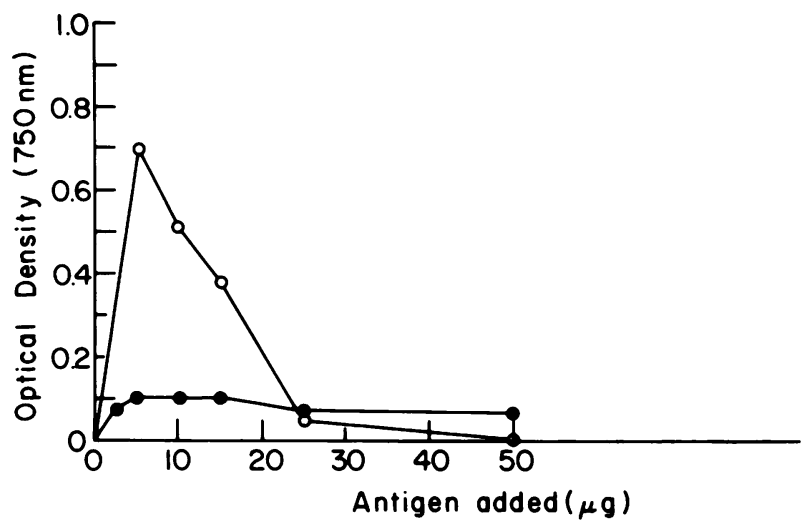

FIGURE 4 Quantitative precipitation of type II group B streptococcal antiserum that was absorbed with a concentration of core antigen at the equivalence point and subsequently reacted with varying concentrations of native antigen (O), and type II serum absorbed with a concentration of native antigen at the equivalence point and subsequently reacted with varying concentrations of core antigen $(\bullet)$.

specific strains grown under conditions of neutral $\mathrm{pH}$. Structural similarities of several sites on these related polysaccharides led to the hypothesis that cross-reactions would exist. Although these heterologous sera did not give capillary precipitin reactions with type II native antigen, all contained some cross-reacting antibodies that were detected by the more sensitive RABA (Table 1). These cross-reactions were not due to contamination of the type II antigen with group polysaccharide because the type II antigen did not react with group B-specific serum and did not contain rhamnose by chemical analysis. The latter sugar has been shown to constitute a major component of the group B, as opposed to type specific, polysaccharide (17).

Clarification of the nature of the cross-reactive determinants required $\mathrm{RABA}$ inhibition with specific antigens (Table II). The determinant expressed in type Ia antiserum that precipitated in the RABA with type II native antigen was inhibited specifically by the Ia native, core, and degalactosylated core polysaccharides. A larger quantity of native Ia polysaccharide than core or degalactosylated core polysaccharide was required for inhibition. The backbone Ib polysaccharide, which has been shown to be identical chemically to the backbone Ia polysaccharide, failed to inhibit the cross-reactive antibodies in type Ia antiserum precipitating with the type II native antigen. Although this cross-reaction could not be inhibited with $>1,000 \mathrm{ng}$ of Ib native polysaccharide, the core and degalactosylated core Ib polysaccharide were efficient inhibitors. The type III native, core, and backbone polysaccharides, all efficiently inhibited the cross-reactive antibodies in Ia antiserum that precipitate with the type II native antigen. Neither the group B polysaccharide nor the type II core polysaccharide inhibited this crossreaction.

Antibodies in serotype III antiserum that precipitated with type II native antigen were inhibited efficiently by the type III native antigen and to a lesser degree by the core type III polysaccharide. No other antigens inhibited this cross-reaction.

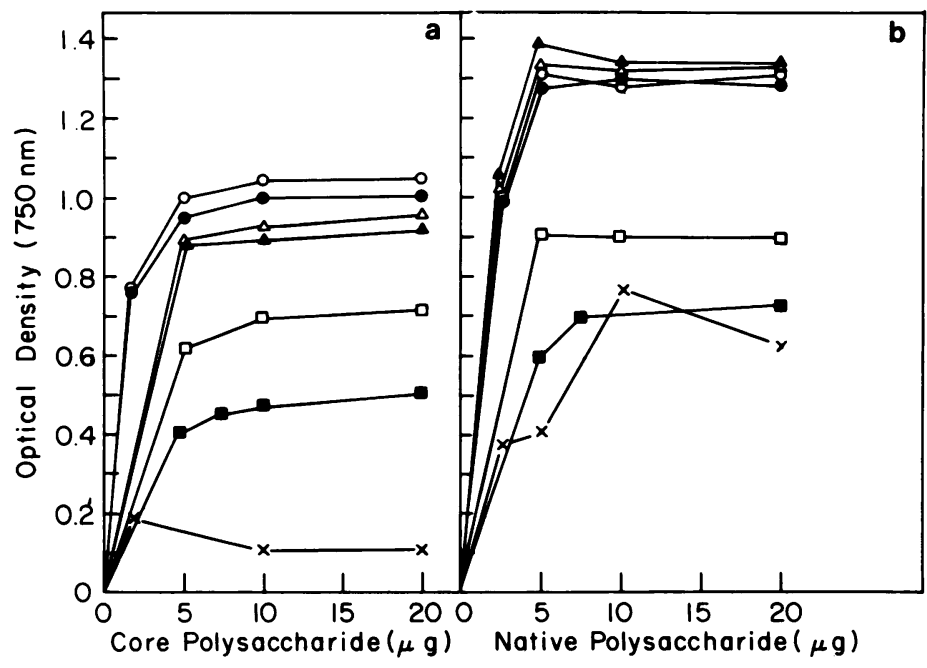

FIgURE 5 Quantitative precipitation inhibition of the reaction between type II group B streptococcal antiserum and the core (a) or native (b) type II polysaccharide by various monosac-

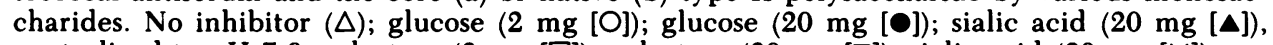
neutralized to $\mathrm{pH} 7.6$; galactose $(2 \mathrm{mg}[\square])$; galactose $(20 \mathrm{mg}[\square])$; sialic acid $(20 \mathrm{mg}[\times])$. 
TABLE II

Inhibition of Antibodies in Various Group B Streptococcal Antisera that React with Type II Native Antigen

\begin{tabular}{|c|c|c|}
\hline \multirow[b]{2}{*}{ Inhibiting antigen } & \multicolumn{2}{|c|}{$\begin{array}{l}\text { Quantity of antigen that gives } \\
50 \% \text { of inhibition }\end{array}$} \\
\hline & la serum & III serum \\
\hline & \multicolumn{2}{|c|}{$n g$} \\
\hline Ia native & 700 & $>1,000$ \\
\hline Ia core & 314 & $>1,000$ \\
\hline Ia degalactosylated core & 284 & $>1,000$ \\
\hline Ib native & $>1,000$ & $>1,000$ \\
\hline Ib core & 280 & $>1,000$ \\
\hline Ib degalactosylated core & $<250$ & $>1,000$ \\
\hline Ib backbone & $>1,000$ & $>1,000$ \\
\hline II native & $<250$ & $<250$ \\
\hline II core & $>1,000$ & $>1,000$ \\
\hline III native & 300 & $<250$ \\
\hline III core & $<250$ & 700 \\
\hline III backbone & $<250$ & $>1,000$ \\
\hline Group B & $>1,000$ & $>1,000$ \\
\hline
\end{tabular}

- Quantity of inhibiting antigen calculated to inhibit the cross-reaction between each serum and type II native polysaccharide by $50 \%$.

All cross-reactions were confirmed with a radiolabeled antigen $\left(\left[{ }^{3} \mathrm{H}\right]\right.$ acetate labeled intrinsically) prepared from a single colony isolate of type II GBS strain 18RS21 to ensure no contamination with other serotypes.

Immunogenicity and safety of native type II polysaccharide in adults. No systemic reactions were observed among the 20 adult subjects immunized with a single $50-\mu \mathrm{g}$ dose of purified native type II polysaccharide. Local reactions at the injection site occurred in three $(15 \%)$ volunteers, consisting of mild erythema $(<2-\mathrm{cm}$ diam) and tenderness that lasted for $<48 \mathrm{~h}$. The antibody responses to the native type II polysaccharide vaccine in these 20 volunteers are summarized in Table III. Since prior studies with native type III polysaccharide vaccine have indicated that preimmunization antibody level is an important determinant of immune response (26), the data were analyzed with this variable in mind. Of the 13 vaccinees with levels of antibody to the type II antigen of $<2 \mu \mathrm{g} / \mathrm{ml}$ in preimmunization sera, $12(92 \%)$ responded with a $>1.0 \mu \mathrm{g} / \mathrm{ml}$ increase in antibody $4 \mathrm{wk}$ later, and the geometric mean concentration of antibody in their sera increased from 1.3 to $10.2 \mu \mathrm{g} / \mathrm{ml}(\mathrm{t}=3.8, P<0.01$; paired $t$ test). Of the seven subjects with $>2 \mu \mathrm{g} / \mathrm{ml}$ of type II antibody in preimmunization serum, each responded to immunization with a $>1 \mu \mathrm{g} / \mathrm{ml}$ increase at $4 \mathrm{wk}$, and the geometric mean antibody in sera from
TABLE III

Result of Immunization of Adults with Type II Group B Streptococcal Vaccine

\begin{tabular}{|c|c|c|c|c|}
\hline \multirow{3}{*}{$\begin{array}{c}\text { Preimmunization } \\
\text { levels }\end{array}$} & \multirow[b]{3}{*}{$n$} & \multicolumn{3}{|c|}{ Antibody to the type II polysaccharide ${ }^{\bullet}$} \\
\hline & & \multirow[b]{2}{*}{ Preimmunization } & \multicolumn{2}{|c|}{ Postimmunization } \\
\hline & & & 4 wk & $2 \mathrm{yr}$ \\
\hline$<2 \mu \mathrm{g} / \mathrm{ml}$ & 13 & $1.3 \pm 0.1$ & $10.2 \pm 3.6$ & $8.0 \pm 3.0 \ddagger$ \\
\hline$\geqq 2 \mu \mathrm{g} / \mathrm{ml}$ & 7 & $5.8 \pm 2.8$ & $29.3 \pm 5.0$ & $22.7 \pm 5.6$ \\
\hline
\end{tabular}

$n$, number of subjects.

- Geometric mean \pm SD $(\mu \mathrm{g} / \mathrm{ml})$.

† Only 11 sera available for testing.

these individuals rose from 5.8 to $29.3 \mu \mathrm{g} / \mathrm{ml}(\mathrm{t}=4.76$, $P<0.01$; paired $t$ test). Vaccine-induced antibody persisted $2 \mathrm{yr}$ after immunization at levels significantly increased over preimmunization values in both groups $(P<0.01$, paired $t$ test $)$.

Since cross-reactions between GBS polysaccharides and hyperimmune type II rabbit antiserum were noted, the possibility that similar cross-reactions might occur in immunized human subjects was investigated. Pre- and 4-wk postimmunization sera of the 20 recipients of native type II polysaccharide were tested by the RABA using radiolabeled native type Ia or type III polysaccharides as antigen. None of the volunteers had any detectable changes in serum antibody concentrations. Conversely, paired sera from subjects immunized with either native type Ia $(n=3)$ or native type III $(n=20)$ polysaccharide vaccines had no detectable change in the concentration of antibody to native type II polysaccharide antigen. Therefore, cross-reactive antibodies could not be demonstrated in human sera.

\section{DISCUSSION}

The continuing prominence of the group B Streptococcus as a cause of neonatal morbidity and mortality has prompted investigative efforts aimed at prevention of infection due to this bacterium. Since susceptibility to invasive infection in mice (4) and in human infants $(6,23,24)$ correlates with low levels of serum antibody directed against the type-specific antigens of GBS, one proposed method for the prevention of neonatal disease is active immunization of child-bearing age women with these polysaccharides. This method presumes immunogenicity and safety of candidate vaccines, induction of IgG class antibody to insure placental transport, and protection by these maternally derived antibodies in neonates susceptible to invasive infection. For type III GBS the first two requirements have been experimentally verified $(24,25)$, although 
protective efficacy remains to be documented. Although little is known about human immunity to other serotypes of GBS, careful immunologic and chemical characterization of their surface antigens has served as a major approach to vaccine development.

Precise knowledge of the structures of specific polysaccharides can allow more definitive identification of their immunodeterminants and insights into the basis of serological cross-reactions involving these antigens. We have explored the structural and immunologic relationships between group B streptococcal antisera and the type II-specific polysaccharide. Jennings et al. (7) have defined structurally the type II native and core polysaccharides. These polysaccharides contain the same monosaccharides found in the type $\mathrm{Ia}, \mathrm{Ib}$, and III GBS polysaccharides but in different molar ratios (Fig. 1). The type III polysaccharide has a single galactose-sialic acid side chain protruding from a trisaccharide backbone, while the type Ia and Ib polysaccharides have disaccharide backbones that are identical. These latter two polysaccharides have similar monosaccharides in their trisaccharide side chain, and their structures differ only in the linkage of galactose to the glucosamine of the side chain.

Earlier studies defined two distinct populations of mouse protective type-specific antibodies directed against different determinants on the capsule of type II GBS organisms $(8,9)$. Antibodies to these determinants were equally protective. One antibody population was directed against an $\mathrm{HCl}$-extracted antigen, which contained galactose, glucose, and glucosamine. Another population of protective antibodies was directed to an acid labile determinant, present on an antigen extracted with cold trichloracetic acid (TCA). This work is the basis for our current understanding of antibody specificity to this GBS serotype.

It is clear now from our structural studies that the acid labile structural determinant described by Lancefield and Freimer was sialic acid; and chemically the cold TCA-extracted type II antigen has enough of this sialic acid remaining to be equivalent antigenically to the native type II polysaccharide.

By precipitation inhibition studies, Freimer (9) found that the monosaccharide, D-galactose, was a specific determinant of the core antigen. We have corroborated his observations and believe that the galactose which was immunodominant is likely to be the side-chain galactose. Galactose is capable of inhibiting a population of the antibodies in type II serum precipitating both the native and core polysaccharides. This finding suggested that this galactose determinant was exposed on both antigens. The requirement for a large excess of galactose for inhibition compared with the amount of precipitating polysaccharide suggested that, although galactose is an inhibitor, the complete immunodeterminant may well be larger than the terminal galactopyranose residue alone.

As with other type-specific polysaccharides (Ia and III), sialic acid appeared to be involved in a native more complete immunodeterminant as indicated by the partial identity (Fig. 2). Although there was a population of antibodies in type II GBS antiserum that was specific for the native determinant, this determinant was not sialic acid alone. "Pseudoinhibition," dependent on $\mathrm{pH}$, was seen by sialic acid in the studies of quantitative precipitation (Fig. 5). Previously, investigators have described sialic acid as an immunodominant sugar of group B streptococcal polysaccharides $(28,29)$. Using neutral conditions, we never have found that sialic acid functions as an independent immunodeterminant. Perhaps this discrepancy is explicable by the pH-dependent phenomenon. Even if sialic acid were part of a determinant on the polysaccharides, it would be unlikely that free sialic acid would be a good inhibitor. Free sialic acid exists preponderantly as the $\beta \mathrm{D}$-anomer in solution (30), whereas the terminal sialic acid residues of the native GBS polysaccharides are in the opposite $\alpha \mathrm{D}$-configuration (7, 13-16).

A series of interesting cross-reactions was described between the type II native antigen and serotype Ia, Ib, and III rabbit antisera. The strongest of these crossreactions occurred with the serotype Ia antiserum. This result was not surprising since both the type Ia and II polysaccharides contain an identical trisaccharide, $[\alpha \mathrm{D}-\mathrm{NeuNAcp}-(2 \rightarrow 3)-\beta \mathrm{D}-\mathrm{Galp}(1 \rightarrow 4)-\beta \mathrm{D}-\mathrm{GlcNAcp}]$ The weakest cross-reaction in contrast, was observed with type Ib antiserum and type II antigen, despite the fact that type Ia and Ib polysaccharides differ only in a single linkage. This linkage difference, however, is sufficient to render the Ib polysaccharide different structurally in the above trisaccharide.

Chemically modified polysaccharides from each of the native antigens were prepared in an attempt to elucidate specific immunodeterminant sites. These structures, found in Fig. 1, were used as inhibitors of the type II native antigen in the RABA. The native type II polysaccharide was an efficient inhibitor at equimolar concentrations, while the type II core polysaccharide was unable to inhibit the cross-reaction between the native type II antigen and these antisera. The type Ia and III antisera inhibition with modified Ia, Ib, and III polysaccharides suggested that the actual site of cross-reaction does not involve the sialic acid determinant of the native antigen, since modified polysaccharides lacking sialic acid also inhibit. Surprisingly, the type II core polysaccharide failed to inhibit cross-reactive antibodies. This discrepancy between the inhibitory ability of native and core type II antigens suggests that sialic acid on the native antigen 
confers a conformationally dependent cross-reactive determinant. Simple removal of that single sugar does change the conformation of the molecule (7) and thereby may alter the immunodeterminant site of cross-reactivity. The loss of sialic acid from the core polysaccharide must be sufficient to rearrange sterically the core molecule in such a way that the immunodeterminants are no longer recognizable. We have previously reported that a conformationally dependent immunodeterminant exists in the type III polysaccharide (16).

Inhibition of the cross-reaction between the type II native antigen and serotype Ia antiserum by modified type Ia-, Ib-, and III-specific polysaccharides suggested that the most likely common immunodeterminant site was $[\beta \mathrm{D}-\mathrm{GlcNAc} p-(1 \rightarrow 3)-\beta \mathrm{D}-\mathrm{Gal} p(1 \rightarrow 4) \beta \mathrm{D}-\mathrm{Glc} p]$. Apparently, the strong conformational forces exerted on these antigens are sufficient to negate differences between branching and nonbranching monosaccharides. The fact that the native Ia polysaccharide was only weakly inhibitory and the native Ib polysaccharide was not inhibitory suggested that, in the native state, the cross-reactive sites were masked by sialic acid. The Ia and Ib core polysaccharides and the degalactosylated core polysaccharides inhibited the crossreaction with serotype Ia antiserum. The results suggest that the sites that define either Ib specificity $(\beta \mathrm{DGal} p-(1 \rightarrow 3)-\beta \mathrm{D}-\mathrm{GlcNAc})$, or Ia specificity $(\beta \mathrm{D}-$ Galp-(1 $\rightarrow 4) \beta \mathrm{D}-\mathrm{GlcNAc} p)$, are not involved in the cross-reaction. The failure of the Ib backbone polysaccharide to inhibit this cross-reaction suggested that the glucosamine residue is an essential part of the immunodeterminant. The type III native, core, and backbone polysaccharides all were capable of inhibiting the cross-reaction between type II native antigen and serotype Ia antiserum. The proposed immunodeterminant $(\beta \mathrm{D}-\mathrm{GlcNAc} p-(1 \rightarrow 3) \beta \mathrm{D}-\mathrm{Gal} p)$, in fact, comprised part of all three inhibitory type III antigens (Fig. 1).

The cross-reaction observed between serotype III antiserum and type II native antigen was only inhibited by the type III native and core polysaccharides. All other polysaccharides failed to inhibit this crossreaction. The cross-reactive determinant in this case was likely to be $(\beta \mathrm{D}-\mathrm{Gal} p-(1 \rightarrow 4)-\beta \mathrm{D}-\mathrm{GlcNAc})$. Interestingly, this disaccharide was also found in the type Ia polysaccharide side chain, yet the type Ia polysaccharide derivatives failed to inhibit the cross-reaction between the serotype III antiserum and the type II native polysaccharide. Two possible explanations for this finding exist. Perhaps a trisaccharide was involved in the cross-reaction of type II antigen with the serotype III antiserum. This trisaccharide was found in the type II native antigen and the type III native and core polysaccharides $(\beta \mathrm{D}-\mathrm{Gal} p-(1 \rightarrow 4)-\beta \mathrm{D}-\mathrm{GlcNAc} p$ $(1 \rightarrow 3)-\beta D-G a l p)$, but not in type Ia polysaccharide.
The other possibility was that this immunodeterminant was also conformationally dependent.

These cross-reactions were not due to contamination by the group B antigen, because $(a)$ that polysaccharide fails to inhibit any cross-reactions; $(b)$ there is no rhamnose in these capsular polysaccharides (rhamnose was immunodominant on the group B polysaccharide); and $(c)$ the type II native antigen fails to react with group B-specific antiserum as measured by the sensitive RABA.

It is interesting that Freimer (9) reported a personal communication from Dr. M. Heidelberger about the finding that the type II $\mathrm{HCl}$ antigen (core) reacted with the type 14 pneumococcal polysaccharide. The type 14 pneumococcal antigen is identical chemically to the core type III GBS antigen $(13,31)$. The basis of the cross-reaction with type 14 pneumococcal polysaccharide and type II GBS antigen may be explained by the same immunodeterminant that was cross-reactive between the type III GBS backbone and type II GBS antigens.

Some polysaccharide vaccines have been shown to be excellent immunogens in humans. Although disease due to type II GBS is less common than type III disease in neonates, type II is the most common serotype responsible for adult disease. It has been our opinion that the type II polysaccharide should be included in formulation of a GBS vaccine.

The native type II polysaccharide given to human volunteers was of large molecular size and was nontoxic in laboratory animals. In the 20 immunized human volunteers, no systemic reactions were observed and local reactions were mild, transient, and infrequent (15\%). $19(95 \%)$ responded with $>1.0 \mu \mathrm{g} / \mathrm{ml}$ increases in specific serum antibody. The response rate to immunization with type II polysaccharide in those individuals who had low preimmunization antibody levels $(92 \%)$ was greater than that reported in adults with low levels of antibody to type III polysaccharide before immunization with that vaccine (73\%) (25). As has been reported for other polysaccharides, antibody levels persisted for at least 2 yr.

The high response rate to this polysaccharide, coupled with the observed cross-reactions between the type-specific polysaccharides and the type II antigen in rabbit antisera suggested the possibility of detecting rises in antibody to other type-specific polysaccharides in humans after immunization with the type II antigen. This hypothesis was attractive particularly because lower response rates have been observed in adults immunized with the Ia or III polysaccharides. ${ }^{2}$

Unfortunately, cross-reactions similar to those found in rabbit antisera were not observed in humans after immunization with the purified type II polysaccharide.

\footnotetext{
${ }^{2}$ Baker, C. J., and D. L. Kasper. Unpublished observations.
} 
Although cross-reactions are attributable to determinants existing on the polysaccharides, it is possible that these determinants were not sufficiently immunogenic to induce cross-reactive antibodies in adults given a single dose of type II polysaccharide.

\section{ACKNOWLEDGMENTS}

We wish to thank Bette Manulick, M.P.H., and Roxanna Krafka, R.N., for their services, and Mrs. Loreen Carr for assistance in the preparation of this manuscript.

This work was supported, in part, by National Institutes of Health contract AI 72538 and National Institutes of Health grant AI 13249.

\section{REFERENCES}

1. Baker, C. J. Group B streptococcal infections. 1980. Adv. Intern. Med. 25:475-501.

2. Wilkinson, H. W., R. R. Facklam, and E. C. Wortham. 1973. Distribution by serological type of group B streptococci isolated from a variety of clinical material over a five-year period (with special reference to neonatal sepsis and meningitis). Infect. Immun. 8:228-235.

3. Wilkinson, H. W. 1978. Analysis of group B streptococcal types associated with disease in human infants and adults. J. Clin. Microbiol. 7:194-201.

4. Lancefield, R. C., M. McCarty, and W. N. Everly. 1975. Multiple mouse-protective antibodies directed against group B streptococci: special reference to antibodies effective against protein antigens. J. Exp. Med. 142:165179.

5. Baltimore, R. S., D. L. Kasper, C. J. Baker, and D. K. Goroff. 1977. Antigenic specificity of opsonophagocytic antibodies in rabbit antisera to group B streptococci. $J$. Immunol. 118:673-678.

6. Hemming, V. G., R. T. Hall, P. G. Rhodes, A. O. Shigeoka, and H. R. Hill. 1976. Assessment of group B streptococcal opsonins in human and rabbit serum by neutrophil chemiluminescence. J. Clin. Invest. 58:13791387.

7. Jennings, H. J., K.-G. Rosell, E. Katzenellenbogen, and D. L. Kasper. 1983. Structural determination of the capsular polysaccharide antigen of type III group B Streptococcus. J. Biol. Chem. 258:1793-1798.

8. Lancefield, R. C., and E. H. Freimer. 1966. Type-specific polysaccharide antigen of group B Streptococcus. J. Hyg. (Cambridge). 64:191-203.

9. Freimer, E. H. 1967. Type-specific polysaccharide antigens of group B streptococci. II. The chemical basis for serological specificity of the type II $\mathrm{HCl}$ antigen. J. Exp. Med. 125:381-392

10. McCarty, M., and R. C. Lancefield. 1955. Variation in the group-specific carbohydrate of group A streptococci. J. Exp. Med. 102:11-35.

11. Lancefield, R. C. 1934. A serological differentiation of specific types of bovine hemolytic streptococci (group B). J. Exp. Med. 59:441-458.

12. Lancefield, R. C. 1938 . Two serological types of group B hemolytic streptoccoci with related, but not identical, type-specific substances. J. Exp. Med. 67:25-40.

13. Jennings, H. J., K.-G. Rosell, and D. L. Kasper. 1980. Structural determination and serology of the native polysaccharide antigen of type III group B Streptococcus. Can. J. Biochem. 58:112-120.

14. Jennings, H. J., K.-G. Rosell, and D. L. Kasper. 1980.
Structure and serology of the native polysaccharide antigen of type Ia group B Streptococcus. Proc. Natl. Acad. Sci. USA. 77:2931-2935.

15. Jennings, H. J., E. Katzenellenbogen, C. Lugowski, and D. L. Kasper. 1983. Structure and serology of the native polysaccharide antigens of types Ia and Ib group B Streptococcus. Biochemistry. 22:1258-1263.

16. Jennings, H. J., C. Lugowski, and D. L. Kasper. 1981. Conformational aspects critical to the immunospecificity of the type III group B streptococcal polysaccharide. Biochemistry. 20:4511-4518.

17. Curtis, S. N., and R. M. Krause. 1964. Antigenic relationships between groups B and G streptococci. J. Exp. Med. 120:629-637.

18. Laurent, T. C., and J. Killander. 1964. A theory of gel filtration and its experimental verification. J. Chromatogr. 14:317-330.

19. Wong, K. H., O. Barrera, A. Sutton, J. May, D. H. Hochstein, J. D. Robbins, J. B. Robbins, P. D. Parkinan, and E. B. Seligmann, Jr. 1977. Standardization and control of meningococcal vaccines, group $\mathrm{A}$ and group $\mathrm{C}$ polysaccharides. J. Biol. Stand. 5:197-215.

20. Ouchterlony, O. 1958. Diffusion-in-gel methods for immunological analysis. Prog. Allergy. 5:1-78.

21. Scheidegger, J. J. 1955. Une micromethode de l'immunoelectrophorese. Int. Arch. Allergy Appl. Immunol. 7:103-110.

22. Kasper, D. L., D. K. Goroff, and C. J. Baker. 1978. Immunochemical characterization of native polysaccharides from group B Streptococcus: the relationship of the type III and group B determinants. J. Immunol. 121:1096-1105.

23. Baker, C. J., and D. L. Kasper. 1976. Correlation of maternal antibody deficiency with susceptibility to neonatal group B streptococcal infection. N. Engl. J. Med. 294:753-756.

24. Baker, C. J., D. L. Kasper, I. Tager, A. Paredes, S. L. Alpert, W. M. McCormack, and D. K. Goroff. 1977. Quantitative determination of antibody to capsular polysaccharide in infection with type III strains of group B Streptococcus. J. Clin. Invest. 59:810-818.

25. Baker, C. J., M. S. Edwards, and D. L. Kasper. 1978. Immunogenicity of polysaccharides from type III group B Streptococcus. J. Clin. Invest. 61:1107-1110.

26. Baker, C. J., D. L. Kasper, M. S. Edwards, and G. Schiffman. 1980. The influence of preimmunization antibody levels on the specificity of the immune response to related polysaccharide antigens. N. Engl. J. Med. 303:173178

27. Hill, A. B. Principles of Medical Statistics. 1971. Oxford University Press, Inc., New York. Ninth ed. 330.

28. Kane, J. A., and W. W. Karakawa. 1977. Multiple polysaccharide antigens of group B Streptococcus, type Ia emphasis on a sialic acid type-specific polysaccharide. J. Immunol. 118:2155-2160.

29. Carey, R. B., T. K. Eisenstein, G. D. Shockman, T. F. Greber, and R. Swenson. 1980. Soluble group- and typespecific antigens from type III group B Streptococcus. Infect. Immun. 28:195-203.

30. Bhattacharjee, A. K., H. J. Jennings, C. P. Kenny, A. Martin, and I. C. Smith. 1975. Structural determination of the sialic acid polysaccharide antigen of Neisseria meningitis serogroups $B$ and $C$ with carbon-13 nuclear magnetic resonance. J. Biol. Chem. 250:1926-1932.

31. Kasper, D. L., C. J. Baker, R. S. Baltimore, J. Crabb, G. Schiffman, and H. J. Jennings. 1979. Immunodeterminant specificity of human immunity to type III group B Streptococcus. J. Exp. Med. 149:327-339. 